\title{
Calculation of First-Level Maintenance Capacity in EMU Depot Based on the EMU Circulation Plan
}

\author{
Jia-nan Tong ${ }^{1, a}$ and Lei $\mathrm{Nie}^{1, \mathrm{~b}}$ and Zhen-huan $\mathrm{He}^{1, \mathrm{c}}$ \\ ${ }^{1}$ School of Traffic and Transportation, Beijing Jiaotong University, \\ Beijing 100044, China \\ a18910125385@163.com, 'Inie@bjtu.edu.cn, czhhe@bjtu.edu.cn
}

Keywords: railway transportation, EMU depot, EMU circulation plan, first-level maintenance capacity, genetic algorithm.

\begin{abstract}
The maintenance capacity of EMU depot(EMUD) not only depends on facilities such as washing tracks and maintenance tracks, but also depends on the EMU circulation plan(ECP). The first-level maintenance of EMU is the most usual one and is almost during night as the special situation of ECP in China, which makes it the key factor of improving EMU operation efficiency and the first-level maintenance capacity of EMUD an essential constraint of the ECP and the timetable. This paper transformed the original problem into flow-shop scheduling problem to establish the integer programming models with ECP as time constraints, and with maximum quantity of EMUs which can be maintained before deadline as the objective function, then used the genetic algorithm to calculate the model. Finally, it was validated by an example in some certain EMUDs of Beijing-Shanghai high-speed railway that the model and algorithm could obtain favorable results for the problem.
\end{abstract}

\section{Introduction}

Currently, as the shortage of maintenance facilities and inefficiency, the EMU maintenance tasks are very tense in most EMUDs of China. The maintenance capacity of the EMUD is also decided by the ECP which is based on the timetable and arranges the EMU operation time, parking stations, train numbers, train routings and EMUD the EMUs belong to. There are five levels of EMU maintenance in China, and the first-level one is the most daily one among them as it is mainly about daily check.

When everyday circulation plan ends, an EMU will transfer to the EMUD nearby from the terminal station to reside or to be maintained, and will transfer back to the station from EMUD to take the circulation task next day. Therefore an EMU can only be maintained after it arrives the EMUD and before it departs the EMUD to guarantee the circulation plan next day, otherwise it should be replaced by one special spare EMU. Thus, the maintenance capacity of EMUD is also related to the ECP.

\section{Description of the Problem}

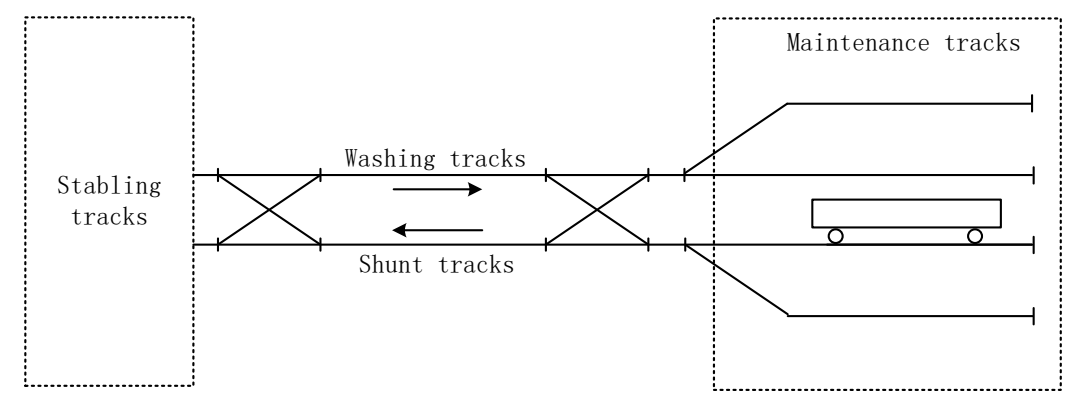

Fig.1. Average layout of EMUD

The layout of an average EMUD contains stabling section, washing section, maintenance section and shunt section as figure 1 shows, and each section has several tracks and undertake different 
maintenance tasks.

The information of EMUs of Jinan EMUD is shown in table 1 as an example, the time an EMU arrives the depot is about $1 \mathrm{~h}$ later than the time it arrives the terminal station and similarly the time it departs the depot is about $1 \mathrm{~h}$ earlier than the time it departs the station next day.

Table 1 The information of EMUs of Jinan EMUD

\begin{tabular}{cccccc}
\hline train no & train type & $\begin{array}{c}\text { time arrive } \\
\text { station }\end{array}$ & $\begin{array}{c}\text { time arrive } \\
\text { depot }\end{array}$ & $\begin{array}{c}\text { time depart } \\
\text { station }\end{array}$ & $\begin{array}{c}\text { time depart } \\
\text { depot }\end{array}$ \\
\hline G178 & CRH380BL & $16: 45$ & $17: 45$ & $7: 10$ & $8: 10$ \\
G64 & CRH380BL & $18: 25$ & $19: 25$ & $6: 50$ & $7: 50$ \\
G7696 & CRH380AL & $20: 59$ & $21: 59$ & $6: 00$ & $7: 00$ \\
G181 & CRH380BL & $21: 09$ & $22: 09$ & $6: 15$ & $7: 15$ \\
D401 & CRH380BL & $21: 57$ & $22: 57$ & $6: 15$ & $7: 15$ \\
D351 & CRH2A & $21: 59$ & $22: 59$ & $6: 05$ & $7: 05$ \\
G7590 & CRH380AL & $22: 12$ & $23: 12$ & $6: 10$ & $7: 10$ \\
D5432 & CRH2A & $22: 21$ & $23: 21$ & $7: 11$ & $8: 11$ \\
D403 & CRH380BL & $22: 25$ & $23: 25$ & $6: 00$ & $7: 00$ \\
G252 & CRH380BL & $22: 57$ & $23: 57$ & $6: 40$ & $7: 40$ \\
D362 & CRH2A & $23: 06$ & $00: 06$ & $6: 05$ & $7: 05$ \\
G183 & CRH380BL & $23: 15$ & $00: 15$ & $5: 42$ & $6: 42$ \\
\hline
\end{tabular}

We can see that the first train arrives the Jinan EMUD at 17:45, and the last one leaves the depot at 7:11, and most of the EMUs are maintained in the span of time from 22:00 to 7:00 next day, which is only 9h. It's the ECP makes the EMUs must maintained in a short span of time in deep night, so we must take it for consideration besides the maintenance facilities.

The import of the problem contains the set of EMUs to be taken maintenance, the set of tracks in each section and the ECP, the output is the quantity of EMUs which can be maintained before the deadline.

\section{The Model}

Let $D=\left\{1,2, \mathrm{~L}, N_{D}\right\}$ denotes the set of all sections( $d=1$ the washing section, $d=2$ the maintenance section and $d=3$ the shunt section), $L_{d}=\left\{1,2, \mathrm{~L}, N_{d}\right\}$ the set of the tracks of section $d, E=\left\{1,2, \mathrm{~L}, N_{E}\right\}$ the set of EMUs. Let $T_{e}^{s}$ and $T_{e}^{e}$ denote the time EMU $e$ arrives and departs EMUD, $t_{e d}$ the minimum duration $e$ should stay on section $d, t_{e d l}^{s}$ and $t_{e d l}^{e}$ the time $e$ arrives and departs the track $l$ of section $d$ (then $t_{\text {edl }}^{e}-t_{\text {edl }}^{s} \geq t_{\text {ed }}$ ), $t_{t}$ the time $e$ shunts between sections, $t_{e}^{s}$ and $t_{e}^{e}$ the start time and finish time of the whole maintenance(then $T_{e}^{s}<t_{e}^{s}$, $\left.t_{e}^{e}<T_{e}^{e}\right)$.

Define two 0-1 decision variables: $x_{\text {edl }}=1$, e occupies track $l$ of section $d$, otherwise; $y_{\text {ecdl }}=1$, $e$ and $c$ both occupy track $l$ of section $d$ and $e$ is the previous EMU of $c$, otherwise. Let $n$ denotes the quantity of EMUs which can be maintained before the deadline. The model is as follows.

$$
\max Z=n
$$

Subject to:

$$
\sum_{l \in L_{d}} x_{e d l}=1, \quad \forall e \in E, \quad d \in D
$$

Equation (2) restricts that each EMU must occupy and can only occupy at most one track at a time on each section.

$$
\left(t_{e d l}^{s}+t_{e d}\right) x_{e d l} \leq t_{e d l}^{e}, \quad \forall e \in E, \quad d \in D, \quad l \in L_{d}
$$

Equation (3) restricts that each EMU must occupy the track for enough time to guarantee the task 
on each section.

$$
x_{e d l} t_{e d l}^{e}+t_{t} \leq \sum_{h \in L_{d+1}} x_{e(d+1) h} t_{e(d+1) h}^{s}, \quad \forall e \in E, \quad d \in D, \quad l \in L_{d}
$$

Equation (4) restricts that only the task of the pre-section is done can the EMU enter the track of next section.

$$
\left(T_{e}^{s}+t_{t}\right) x_{e d l} \leq t_{e d l}^{s}, \quad \forall e \in E, \quad d=1, \quad l \in L_{d}
$$

Equation (5) restricts that the time the maintenance of one EMU starts is no earlier than the time it arrives the EMUD.

$$
\left(t_{e d l}^{e}+t_{t}\right) x_{\text {edl }} \leq T_{e}^{e}, \quad \forall e \in E, \quad d=N_{D}, \quad l \in L_{d}
$$

Equation (6) restricts that the time the maintenance of one EMU ends is no later than the time it departs the EMUD.

$$
t_{e d l}^{e}+t_{t} \leq t_{c d l}^{s}+M\left(1-y_{\text {ecdl }}\right)
$$

Equation (7) is time and space constraint that only the previous EMU leaves the track can the next one moves in, $M$ is a large enough number.

\section{The Algorithm}

The flow-shop problem belongs to NP-hard problems, which are difficult to be solved by traditional mathematical programming approaches. This paper used a solving algorithm based on the self-adaptive genetic algorithm for the model.

The Code of Gene. Let $S$ denotes the number of sections, $N$ the number of EMUs, then create a $S \times N$ code matrix with the element $a_{i j}$ which is a random number in $\left(1, M_{i}+1\right)$, and denotes that EMU $j$ will take the track $\operatorname{int}\left(a_{i j}\right)$ of section $i, M_{i}$ denotes the quantity of the tracks of section $i$.

Fitness Function. This paper designed the fitness function with the inverse of the object function as follows.

$$
f=1 / \max Z
$$

Selection. This paper used roulette method to select chromosomes from the population, and the probability of one individual being selected is designed as follow.

$$
P_{s}=f / \sum_{i=1}^{N} f_{i}
$$

Crossover. This paper used the self-adaptive genetic algorithm, the crossover probability $P_{c}$ was designed as follows.

$$
P_{c}=\left\{\begin{array}{cl}
\frac{k_{1}\left(f_{\max }-f^{\prime}\right)}{f_{\max }-f_{\text {avg }}} & f^{\prime}>f_{\text {avg }} \\
k_{2} & f^{\prime} \leq f_{\text {avg }}
\end{array}\right.
$$

$f_{\max }$ denotes the maximum fitness value of all individuals, $f_{\text {avg }}$ denotes the average fitness value of all individuals, $f^{\prime}$ denotes the larger fitness value of the two individuals to be selected, $k_{1}$ and $k_{2}$ denote the constants in $(0,1)$.

The Mutation. The steps of the mutation are as follows: $d=\operatorname{rand}(1)$; if $d<P_{m}$, then $a_{i j}^{\prime}=\left(M_{i}+1-a_{i j}\right) \times$ rand $(1)+a_{i j}$; else $a_{i j}^{\prime}=\left(a_{i j}-1\right) \times$ rand $(1)+1$.

\section{Computational Experiment}

The model and algorithm were validated by an example in some certain EMUDs of Beijing-Shanghai high-speed railway, the information of each EMUD is shown in table 2. The wash time is 30min per EMU, the shunt time is $10 \mathrm{~min}$ per EMU, and the transfer time between sections is 
5min per EMU.

Table 2 Information of Each EMUD

\begin{tabular}{cccccc}
\hline EMUD & $\begin{array}{c}\text { quantity of } \\
\text { washing } \\
\text { tracks }\end{array}$ & $\begin{array}{c}\text { quantity of } \\
\text { maintenance } \\
\text { tracks }\end{array}$ & $\begin{array}{c}\text { quantity of } \\
\text { shunt } \\
\text { tracks }\end{array}$ & $\begin{array}{c}\text { maintenance } \\
\text { time per } \\
\text { EMU/h }\end{array}$ & $\begin{array}{c}\text { actual quantity of } \\
\text { maintained } \\
\text { EMUs per day }\end{array}$ \\
\hline Beijing South & 2 & 12 & 1 & 3.5 & 56 \\
Jinan & 1 & 6 & 1 & 4 & 17 \\
Nanjing South & 1 & 8 & 1 & 3 & 24 \\
Hongqiao & 3 & 14 & 1 & 3 & 60 \\
\hline
\end{tabular}

We can calculate the time EMUs arrive and depart a depot by the ECP, and the temporal distribution of EMUs in each depot is shown in figure 2. Most of the EMUs arrive the depot in deep night and depart the depot in early morning next day.

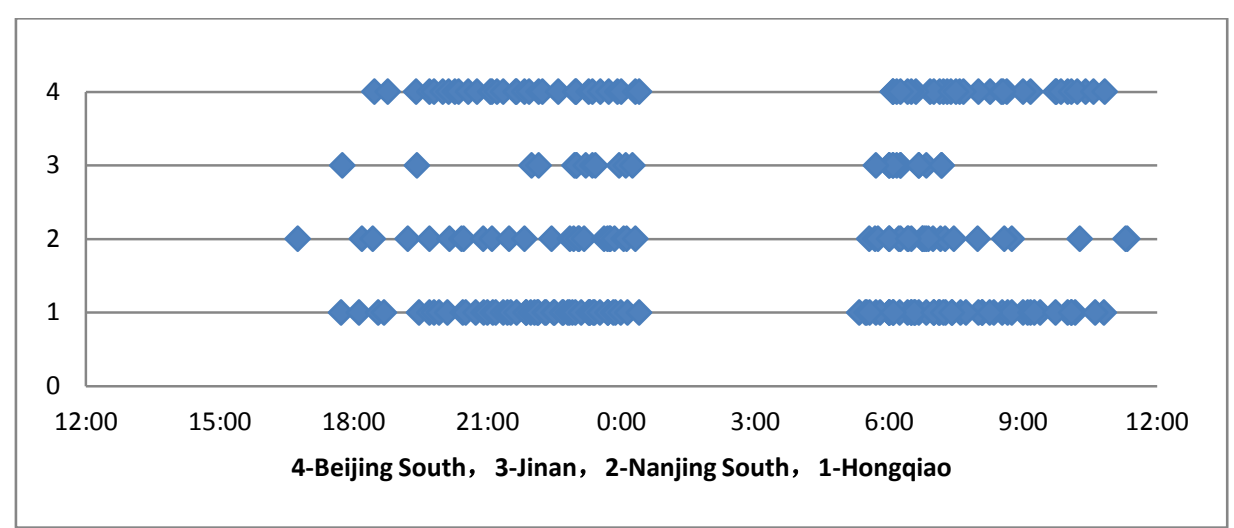

Fig. 2. Time the EMUs arrive and depart each depot

After many experiments, we set the iterations $M=500$, the quantity of population $P=30$, the crossover probability $P_{c}=0.8$, the mutation probability $P_{m}=0.1$, and $k_{1}=k_{2}=0.5$ to calculate the model, and the results for the problem is shown in figure 3.

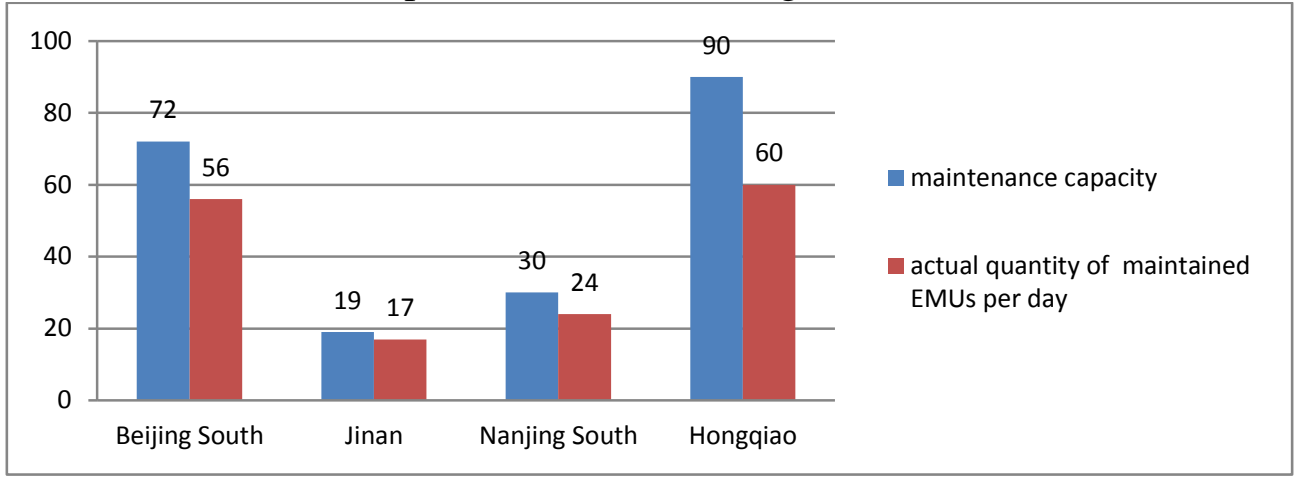

Fig. 3. The Results of the Problem

It shows that the calculated maintenance capacity is larger than the actual quantity of maintained EMUs per day, because the actual maintenance situation is more complex and extra time will be taken for trouble eliminating. Figure 2 also shows that the maintenance tasks of Jinan EMUD and Nanjing South EMUD are quite tense, more washing tracks and maintenance tracks should be constructed and the efficiency of maintenance should be increased.

\section{Literature References}

Some scholars has studied on the problem of the EMU maintenance in EMUD. [1] and [2] estimated the maintenance time of a certain number of EMUs based on maintenance facilities' quantities and the duration of each EMU in each section. [3] studied on the EMU shunt plan of first-level and second-level maintenance in EMUD, transformed the original problem into a job-shop scheduling problem, designed a solving algorithm based on the MAX-MIN ant system for the model. [4] studied on the operation plan of the stabling track in EMUD, established an 0-1 
planning optimization model for stabling track with the fixed EMU holding time of the stabling track as precondition and designed a simulated annealing algorithm based on $\mathrm{k}$ rejecting neighborhood of maximum EMU stabling track operation plan to solve the problem.

In conclusion, current studies rarely calculate the maintenance capacity of EMUD directly, and mostly estimate based on the maintenance facilities' quantities without considering the factors of ECP.

\section{Summary}

Currently, as the shortage of maintenance facilities in most EMUDs, the EMU maintenance tasks are very tense in China, the first-level maintenance capacity of EMUD is instructive to the establishment of EMUD and to make the plan of EMU operations. This paper transformed the original problem into flow-shop scheduling problem to establish the integer programming models and used the genetic algorithm to calculate the maintenance capacity of EMUD, the results show that the model and algorithm could obtain favorable results for the problem.

\section{Acknowledgments}

This study was supported by grants from the National Natural Science Foundation of China(U1434207), the Project of China Railway Corporation(2013X014-C) and the Project of Ministry of Transport of the People’s Republic of China(2015-2-3).

\section{References}

[1] LIU C Q, WU Z Q, LI Q et al. Analysis and countermeasures of Beijing EMUD[J]. Railway transport and economy, 2012, 34(7): 88-90.

[2] LI B. Optimization of the first and second maintenance capacity of EMUD[J]. Railway Topics, 2008, 12(1): 28-30.

[3] WANG Z K, SHI T Y, ZHANG W J et al. Model and algorithm for optimized formulation of scheduled shunting operation plans of electric multiple units depots[J]. Journal of the China railway society, 2013, 35(8): 1-9.

[4] ZHANG W J, SHI T Y, CHEN Y. Model and algorithm for optimized formulation of operation plans of storage tracks[J]. China railway science, 2013, 34(1): 121-25.

[5] Reeves C R. A genetic algorithm for flow shop sequencing[J]. Computers and Operations Research, 1995, 22(1): 5-13. 